\title{
atrancust
}

Revista Educación

ISSN: 0379-7082

ISSN: 2215-2644

revedu@gmail.com

Universidad de Costa Rica

Costa Rica

\section{Compromiso y motivación escolar: Una discusión conceptual}

Sandoval-Muñoz, María José; Mayorga-Muñoz, Cecilia Janette; Elgueta-Sepúlveda, Herman Eduardo; Soto-Higuera, Abel Isidoro; Viveros-Lopomo, Jacqueline; Riquelme Sandoval, Sandra Verónica

Compromiso y motivación escolar: Una discusión conceptual ${ }^{1}$

Revista Educación, vol. 42, núm. 2, 2018

Universidad de Costa Rica, Costa Rica

Disponible en: http://www.redalyc.org/articulo.oa?id=44055139004

DOI: https://doi.org/10.15517/revedu.v42i2.23471

Esta obra está bajo una Licencia Creative Commons Atribución-NoComercial-SinDerivar 3.0 Internacional. 


\section{Compromiso y motivación escolar: Una discusión conceptual ${ }^{1}$}

School Engagement and Motivation: A Conceptual Discussion

María José Sandoval-Muñoz [2] Universidad de Magallanes, Chile mariajose.sandoval@ufrontera.cl

Cecilia Janette Mayorga-Muñoz [3] Universidad de La Frontera, Chile cecilia.mayorga@ufrontera.cl

Herman Eduardo Elgueta-Sepúlveda [4]

Universidad de Magallanes, Chile

herman.elgueta@ufrontera.cl

Abel Isidoro Soto-Higuera [5]

Universidad de La Frontera, Chile

abel.soto@ufrontera.cl

Jacqueline Viveros-Lopomo [6]

Universidad de La Frontera, Chile

jacqueline.viveros@ufrontera.cl

Sandra Verónica Riquelme Sandoval [7]

Universidad de La Frontera, Chile

sandra.sandoval@ufrontera.cl
DOI: https://doi.org/10.15517/revedu.v42i2.23471 Redalyc: http://www.redalyc.org/articulo.oa?id=44055139004

\section{ReSUMEN:}

El presente trabajo tiene como objetivo ahondar en los conceptos de "motivación escolar" y "compromiso escolar" en el contexto de los procesos educativos, examina en qué medida las conceptualizaciones que ofrece la bibliografía anglosajona son compatibles o están representadas en la iberoamericana, con miras a precisar con mayor detalle sus diferencias para favorecer futuras investigaciones o intervenciones. Para ello, se llevó a cabo una revisión teórica que pone en discusión los avances en el análisis de ambos conceptos, se descubrieron similitudes y diferencias en las dimensiones constitutivas de cada una. La de origen iberoamericano trata ambos conceptos como homologables entre sí y produce confusiones conceptuales. Esta revisión permite precisar las diferencias, lo que puede ser de utilidad para favorecer futuras investigaciones o intervenciones.

Palabras Clave: Motivación escolar, compromiso escolar, proceso educativo.

\section{ABSTRACT:}

\section{NOTAS DE AUTOR}

[2] Master in Education, University of Leeds, UK

[3] Doctora en Ciencias Humanas y Sociales: Estudio y Tratamiento de las Relaciones Familiares, Universidad Pontificia Comillas Madrid, España,

[4] Doctor of Philosophy, University of Sheffield, UK:

[5] Magister en Ciencias Sociales Aplicadas,

[6] Doctor en Ciencias de la Educación Universidad de La Frontera, Departamento de Educación

[7] Doctora en Procesos Sociales y Políticos en América Latina, Universidad de Artes y Ciencias Sociales, Chile. 
The present research work aims at gaining understanding of what it is referred as "motivation" and "engagement" in the context of educational processes considering points of consensus and debate in the specialized literature. The objective was examining how far the definitions that have been proposed by authors from English-speaking contexts are compatible or have been represented in writings from Iberoamerican context attempting to clarify how are these two constructs related to each other. A review was performed focusing on manuscripts that discussed advances in these subjects. The main conclusions from this revision highlight the similarity of some definitions for both concepts. It is shown that both concepts are being treated as equivalent to each other, enabling potential conceptual confusions. The need for further debating about the relationship between both kinds of processes aiming at achieving higher clarity regarding the differences between the concepts to help future research and interventions is proposed.

KEYWORDS: school motivation, school engagement, education process.

\section{INTRODUCCIón}

Numerosos esfuerzos investigativos en educación han buscado identificar y estudiar factores que incidirían positivamente en las trayectorias educativas de niños, niñas y adolescentes, facilitando sus procesos de aprendizaje o protegiéndoles de problemáticas como la deserción o el fracaso escolar. Aunque el amplio espectro de investigaciones demuestra que estos procesos estarían afectados por condicionantes a lo largo de múltiples dimensiones que incluyen temas socio-estructurales, culturales, comunitarios, familiares, curriculares y escolares, una porción de estudios se ha enfocado en los procesos psicológicos vividos por el estudiantado que influyen en su aprendizaje y sus posibilidades de logro escolar.

En un intento por resumir hallazgos entregados por diversas investigaciones, Hattie (2013) reanalizó los datos de más de 800 meta-análisis en educación, entre los cuales 29 se enfocaron de alguna manera en examinar relaciones entre el logro académico y variables referidas a procesos psicológicos de estudiantes (implican datos de un total de 1446 estudios y más de 1 millón de estudiantes). Entre las variables examinadas en este sub-grupo de meta-análisis, aquellas que demostraron en términos estadísticos una relación más fuerte con el logro académico fueron las referidas a "motivación” y "compromiso", i.e. en inglés, motivation y engagement, demostraron un tamaño del efecto (ambas $\mathrm{d}=.48$ ) mayor al de otras variables de carácter psicológico como serían la personalidad, el autoconcepto, la creatividad, la disminución de la ansiedad o las actitudes hacia ciertas asignaturas.

Para abordar esta tarea se revisaron conceptualizaciones propuestas por estudios de origen anglosajón y de origen iberoamericano. Aunque en ningún caso proponemos que la conceptualización que pueda ofrecer el mundo académico anglosajón sea necesariamente superior, consideramos importante reconocer que dicho cuerpo suele conformar la corriente principal disciplinaria y de investigación en educación (i.e. mainstream), por lo que las investigaciones en el área no deberían "pasar por alto" sus aportes (especialmente si fuese necesario rebatir sus posturas).

En cuanto a "motivación", García y Daménech (1997) afirman que "la motivación es la palanca que mueve toda conducta, lo que permite provocar cambios tanto a nivel escolar como de la vida en general” ( $p$. 1). Por otro lado, Peralbo, Sánchez y Simón (1986) nos recuerdan que la motivación no es observable, sino un supuesto que se construye a partir de los resultados observados (Godefroid 1991; Legendre, 1993; Raynal; Rieunier, 2014); por tanto, es una inferencia que hacemos a partir de manifestaciones conductuales. Asimismo, definiciones similares se han planteado respecto a lo que refiere a "compromiso" (e.g. Fredricks, Blumenfeld y Paris 2004), que es ampliamente mencionado en la bibliografía anglosajona, pero escasamente referido en textos de origen iberoamericano. Esto da cuenta de que los conceptos podrían estar siendo utilizados de forma sobrepuesta o cruzada, eventualmente llevando a confusiones teóricas. 


\section{Compromiso escolar}

El "compromiso" es considerado actualmente como un relevante predictor del desempeño académico que podría alcanzar el estudiantado (Appleton, Christenson y Furlong, 2008; Shernoff y Schmidt, 2008; Weiss, Carolan, Baker-Smith, 2010), como un antecedente para la obtención de bajos indicadores de deserción escolar y del grado de involucramiento que este tiene en relación con su escuela y el quehacer educativo (Finn, 1989; Jimerson, Campos y Greif, 2003).

Desde el ámbito de la intervención, De Toro et al. (2016) plantean que existe una amplia variedad de programas y prácticas en el contexto escolar que abordan el compromiso y que poseen resultados positivos en su aplicación. Sin embargo, dichos contextos se encuentran mayoritariamente en ambientes anglosajones y existe nula evidencia de intervenciones en contextos iberoamericanos.

Considerando la importancia que se le atribuye a este concepto, es fundamental ahondar para comprender a lo que se refiere este "compromiso". Aunar criterios sobre lo que se entiende por dicho concepto no ha estado exento de múltiples definiciones alternativas que en ocasiones son ambiguas o contradictorias, principalmente en cuanto a variables o dimensiones necesarias de ser consideradas a la hora de medir este constructo (Corso, Bundick, Quaglia, Haywood, 2013; Hart, Stewart, Jimerson, 2011; Hazel, Vazirabadi, Gallagher, 2013).

Al hablar de compromiso, algunos autores y autoras han optado por utilizar el término "compromiso del estudiante" poniendo el foco en el estudiantado y en la experiencia interna de este mismo (Appleton et al., 2008; Christenson, Reschley y Wylie, 2012). Por otro lado, otros textos prefieren referirse a compromiso escolar y destacan el énfasis no solo en el estudiantado, sino que además se deben considerar las variables contextuales como elementos claves para su conceptualización (Fredricks, Blumenfeld, Friedel y Paris, 2003; Jimerson et al., 2003).

En revisiones anteriores, se marca una clara tendencia a utilizar la definición propuesta por Fredricks et al. (2004) que entiende este concepto como un constructo multidimensional conformado por distintas, pero integradas dimensiones o componentes que interactúan entre sí, incluyendo aspectos conductuales, emocionales/sociales y cognitivos.

Más específicamente, el compromiso escolar conductual se refiere a las conductas observables dentro del contexto escolar, tales como participación en actividades académicas y extraprogramáticas, asistencia a clases, buen comportamiento, entre otros (Finn y Voelkl, 1993; Green, Rhodes, Hirsch, Suárez-Orozco y Camic, 2008; Marks, 2000). Por otro lado, el compromiso escolar cognitivo implicaría ir más allá de una identificación de conductas, supone el análisis de los eventos que ocurren a nivel interno del sujeto, el esfuerzo que estudiantes realizan en un determinado aprendizaje, la calidad o el tipo de procesamiento de información, el uso de estrategias metacognitivas como la autorregulación del aprendizaje, entre otras (Shernoff, 2013). Por último, el compromiso escolar emocional está referido a los lazos afectivos que el estudiantado establece dentro de este contexto, no tan solo con sus pares, sino también con su profesorado (Faria, Freire, Galväo, Reis, Baptista, 2012). En efecto, este tipo de compromiso daría cuenta de toda experiencia que implique el involucramiento de emociones tanto positivas como negativas hacia las relaciones o actividades que estén dentro de un contexto escolar (Finn, 1989; Voelkl, 1997).

En esta misma línea, numerosos manuscritos plantean la definición de compromiso escolar como un metaconstructo que se sobrepondría a concepciones similares, tales como conexión y lazos con la escuela, actitud positiva y apego hacia ella o fuerte unión entre estudiante y escuela, en las distintas dimensiones ya descritas anteriormente (Hart, et al, 2011; Veiga, 2012, Veiga, 2013).

Siguiendo este enfoque multidimensional, resultaría coherente entender compromiso escolar como un fenómeno biopsicosocial (Hazel, et al., 2013) que se relaciona no solo con el desarrollo esperado del estudiantado, sino también, con el contexto en el que ocurre dicho desarrollo, el que tiene normas, reglas y valores específicos a los que debe adaptarse para poder alcanzar y desarrollar este compromiso. 
Por otro lado, es necesario destacar lo que Shernoff (2013) plantea como críticas a las definiciones ya señaladas. De acuerdo con dicho autor, tales definiciones estarían relacionadas principalmente con lo que se espera de un o una estudiante "ideal". Es decir, entender compromiso desde el área conductual, sería evaluar al estudiantado de acuerdo con actividades que la escuela espera de él, como serían la alta participación en las actividades, el término de una tarea en los tiempos estimados o la participación activa con su grupo de trabajo.

Sin embargo, no se estaría analizando si esto es una representación del compromiso del estudiantado o es una simple muestra de complacencia a la figura de autoridad (Shernoff, 2013). Este comportamiento variaría o asumiría características particulares, dependiendo del contexto escolar del estudiantado. Mirado desde otro punto, compromiso escolar estaría siendo un concepto altamente deseado, difícil de alcanzar y mantener de manera constante a través del tiempo.

Si el consenso actual apunta a considerarlo como un elemento principalmente psicológico, que implica esfuerzo, entendimiento y logro del conocimiento esperado (Newmann, Wehlage y Lamborn, 1992), debe ser entendido como un concepto que requiere de un individuo activo, involucrado en una meta específica, que implique persistencia, lo que necesita de cada estudiante el deseo de interactuar con el entorno (Furrer y Skinner, 2003).

Por lo tanto, un aspecto que resulta altamente coherente en las definiciones planteadas es el hecho de que compromiso escolar presenta un componente interno asociado a las dimensiones presentadas por Fredricks et al. (2004), que instala el foco en el estudiantado, pero que a la vez este desarrolla su experiencia en un contexto determinado. Contexto que presenta características específicas y que implica, además, interacción, intercambio de información, que cambia en la medida que interactúa con el estudiantado, lo cual nos permite analizarlo desde un punto de vista ecológico (Shernoff, 2013).

En la búsqueda de la clarificación del concepto parece pertinente remontarse al origen de este mismo. Al respecto, señalar que este concepto o idea se atribuye a Csikszentmihalyi (1990), quien lo definió como una "experiencia de fluidez" (i.e. flow experience en inglés), entendiéndolo como un estado óptimo (cognitivo y emocional) que alcanza un individuo y que le permite estar conectado completamente con una tarea, deja de lado cualquier tipo de distracción, conciencia de tiempo, etc.

Tomando por base lo propuesto por Csikszentmihalyi, para Shernoff (2013), compromiso escolar se define como una simultánea experiencia de concentración, interés y disfrute intenso del estudiantado en una tarea específica. De acuerdo con lo señalado por el autor, se desprenderían dos razones que justificarían su preferencia por tal conceptualización. Primero, no apunta a cómo el estudiantado debería pensar, sentir o actuar en un contexto escolar. De hecho, la definición no específica el contexto, lo cual permite comprender que el compromiso escolar no solo ocurre dentro de una escuela, sino que debería desarrollarse también en contextos más informales.

En segundo lugar, dicho autor señala que el compromiso escolar estaría basado completamente en la experiencia estudiantil, por lo tanto, debería ser considerado como una "experiencia de aprendizaje", teniendo un valor en sí mismo, y no tan solo como un predictor. En este sentido, es relevante destacar que, a pesar de ciertas diferencias o sutilezas en las definiciones planteadas, se resalta la idea de definir compromiso escolar asociado a componentes cognitivos frente a una tarea específica, como también a elementos altamente afectivos o emocionales que estarían determinando el interés o la energía necesaria que requeriría el estudiantado dentro de un determinado contexto para lograr el desarrollo exitoso del aprendizaje. En consecuencia, al intervenir el ambiente escolar en el que se desarrolla el compromiso, este sería una variable adaptable que se puede modificar (Dotterer, McHale, Crouter, 2007).

\section{Motivación escolar}

Respecto a la motivación escolar, algunos estudios (Beltrán, 1993; Bueno, 1995; McClelland, 1989) la conceptualizan como un proceso que activa la conducta, la direcciona y la mantiene en el tiempo. Debido 
a la intencionalidad que genera en la conducta del estudiantado, su autopercepción, sus expectativas y las metas que quiere alcanzar, constituyen los elementos centrales que guían su comportamiento en el contexto escolar. Otras investigaciones (Archambault y Chouinard,1996; Tardif, 1992) agregan además que no tan solo impulsa al estudiante a adquirir un compromiso activo frente a su aprendizaje, sino que, además, permite perseverar ante los posibles obstáculos y lograr de esta forma los objetivos propuestos.

Similar propuesta plantean Martínez (2011a) y Valle y González (1996) cuando se refieren a ella como una competencia que comporta una serie de procesos cognitivos, afectivos, conductuales y relacionales que orientan al estudiantado en dirección a sus logros, a través del desarrollo de conductas sistemáticas y persistentes en el tiempo, condicionadas eso sí, por el contexto social en el que ocurre el proceso. En suma, los distintos conceptos coinciden en referirse a un conjunto de procesos personales y de contextos que estimulan o limitan la conducta del estudiantado hacia el logro de ciertos objetivos y deseos en el medio escolar.

Estudios previos sobre los componentes asociados a procesos cognitivos y afectivos relevan el papel determinante que juega en la motivación la interrelación de factores que incluyen el autoconcepto, atribución causal, o metas de aprendizaje (Núñez y Gonzalez-Pumariega, 1996; Tardif, 1992; Viau, 1997).

Por su parte, Pintrich y De Groot (1990) identifican tres dimensiones constitutivas de la motivación: las expectativas, las metas y la afectividad con respecto a la tarea. Las primeras se refieren a las creencias y resultados que espera obtener el estudiantado cuando hace una tarea. El logro de las metas que se propone estaría condicionado por la importancia que le atribuyen a la actividad y, por último, el vínculo afectivoemocional que tienen con ella.

Coincidentemente, Núñez y González-Pumariega (1996) plantea que la motivación es un proceso múltiple, que involucra variados componentes que ninguna de las actuales propuestas teóricas ha logrado integrar, es una tarea pendiente precisar y clarificar qué elementos o factores específicos son parte del proceso de motivación. Independientemente de que muchas de ellas explican la motivación como conductas orientadas hacia una meta que van siendo modeladas por procesos sociales y cognitivos (Furrer y Skinner, 2003; Wentzel, 2005), en su mayoría, han puesto el foco en relacionar la motivación con el rendimiento académico y en consecuencia relevar los factores personales involucrados en dicho proceso.

Tales apreciaciones se fundamentan en estudios previos que muestran la motivación como un importante predictor del resultado académico (Inglés et al., 2009; Steinmayr y Spinath, 2009) y, en consecuencia, un dato relevante para las acciones preventivas orientadas a disminuir el fracaso escolar y la deserción escolar temprana (Alderman, 2008; Meece, Anderman y Anderman, 2006; Valle et al., 2010), pues es el activador de la generación de nuevas estrategias de aprendizaje en el estudiantado, que mejora su rendimiento académico (Covington, 2000), por lo que tener o no tener motivación resulta un factor relevante a la hora de enfrentar dificultades en el proceso educativo y, en consecuencia, condiciona el éxito en los resultados académicos.

Los conceptos expuestos dejan entrever dos líneas o enfoques considerados en su elaboración: la primera que define la motivación en función de factores personales del individuo; una segunda que busca poner el foco en los factores ambientales o de contexto. Sin embargo, como se ha señalado precedentemente, la motivación académica ha sido abordada mayoritariamente como una variable de carácter personal, asociada a componentes o procesos internos del individuo, desestimando la posible influencia en ella de aspectos relativos a los contextos sociales de la escuela, la familia o la comunidad.

Al respecto, dentro de los factores contextuales, la familia y el ambiente social jugarían un papel preponderante. Estudios como los de Torío (2004) enfatizan que se espera que el contexto familiar, como agente de socialización, promueva el óptimo desarrollo de los hijos e hijas a nivel general y potencie en ellos y ellas actitudes positivas hacia el estudio (Asensio, 1994; Marjoribanks, 2003), lo que, sin duda, contribuiría a mejorar la motivación de niños, niñas y adolescentes. En esta misma línea, Broc (2000) constató que una variable predictora importante sobre la autoestima global de la persona joven es el apoyo que recibe por parte de sus familias, pares de aula y profesorado. Lo que muestra la relación y mutua influencia entre el 
mundo familiar y escolar, dos realidades en las que se mueve cotidianamente el individuo en estas etapas de su desarrollo.

Algunas teorías actuales sobre motivación consideran los factores contextuales como espacios para vivenciar experiencias y oportunidades para obtener nuevos conocimientos. Dichos factores pueden estimular o desalentar al estudiantado a darle sentido a una experiencia concreta, enfatizándose de este modo el rol mediador del ambiente en el aprendizaje, ya que como señala Hickey (2003), los valores, patrones comportamentales y criterios preestablecidos en esos entornos pueden reforzar la motivación al aprendizaje y debieran permitir una participación significativa, lo que implica facilitar el aumento de interacciones personales satisfactorias.

\section{Análisis de la relación o sobreposición entre ambos conceptos}

\subsection{Similitudy diferenciación de ambos conceptos}

Desde la descripción y análisis planteado sobre ambos conceptos, nos sumamos al debate que actualmente está presente en la bibliografía de origen anglosajón, respecto a la relación que existe entre compromiso y motivación escolar. Debate ausente o escasamente abordado en los estudios iberoamericanos.

Los argumentos revisados dan cuenta del intento por tratar de diferenciar ambos conceptos, considerando por un lado el compromiso escolar como esta energía que permite la acción, la interacción o conexión del estudiantado con una tarea determinada (Rusell, Ainley y Frydenberg, 2005) como también la evidencia de la realización de dicha tarea que implica, a su vez, la presencia de motivación a la base (Skinner, Kindermann, Connel y Wellborn, 2009; Wentzel, Wigfield y Miele 2009). La suficiente motivación como para superar los obstáculos que se pueden presentar en el camino, al permitir continuidad en la acción a realizar, hasta el logro de la meta o finalización de la tarea (Robson, Albanese, Harrison y Sanders, 2014).

Por otro lado, Maehr y Meyer (1997) consideran el concepto de motivación en términos de la dirección que puede tomar el desarrollo de la actividad, como también la intensidad y la calidad de la energía puesta. Desde este planteamiento, la motivación se entendería como el volante y la caja de cambios de un automóvil, lo cual permitiría aumentar o disminuir la potencia de una acción a ejecutar, y donde el compromiso estaría cumpliendo el rol del auto, que ejecuta la acción.

Skinner et al. (2009) describen la motivación en términos de procesos psicológicos, como autonomía, sentido de pertenencia (Furrer y Skinner, 2003) y capacidad del individuo para desarrollar determinada tarea (Furlong y Christenson, 2008). Sin embargo, también plantean que la motivación es un requisito necesario, pero no suficiente para la realización del compromiso. En este sentido, sugieren considerar la motivación escolar como un elemento de importancia que sería parte de la formación o el desarrollo del compromiso, pues este último comporta la presencia de otras variables.

\subsection{Factores constitutivos del concepto de compromiso y de motivación}

Como se observa en apartados anteriores, no existe una clara diferenciación en la conceptualización entre los constructos de motivación escolar y compromiso escolar, pues ambos coinciden en reconocer ciertas características y capacidades del sujeto como propias. En este sentido encontramos algunos factores comunes en ambas definiciones: 


\subsubsection{Factor procesos cognitivos}

Dentro de los procesos cognitivos, destacan dos elementos que cobran relevancia en ambos conceptos, pues se refieren a procesos internos observables en la conducta del estudiantado, que son los que se relacionan con habilidades metacognitivas como autorregulación y autoeficacia, elementos necesarios para el desarrollo de una tarea o logro de una meta académica (Pintrich, Schunk y Luque, 2006). Tal como señala Zimmerman (1999), el compromiso de tipo cognitivo tendría su origen en un nivel interno, dado que se relaciona con el esfuerzo que el estudiantado realiza para incorporar nuevos aprendizajes y los procesos intermedios que debe realizar para ello.

En este sentido, el concepto de autorregulación sería un elemento importante dentro del compromiso cognitivo, ya que apunta a la manera en que el estudiantado enfrenta las tareas de aprendizaje o bien las estrategias que utiliza para lograr con éxito la acción propuesta (Martínez, 2011b; Zimmerman, 1998).

García, Barbero, Ávila y García (2003), Torrano y González (2004) señalan que el estudiantado que se autorregula y que se motiva a sí mismo, se caracteriza por el uso de estrategias cognitivas, por regularse emocionalmente y controlar la ansiedad, por tener sentido de autoeficacia y por lograr gestionar sus recursos para ir más allá de las actividades solicitadas, como también de participar en tareas vinculadas a sus propios intereses. Así, la autorregulación determina, en gran medida, la motivación interna frente al desarrollo de tareas de aprendizaje, y afecta, ya sea de manera positiva o negativa, el desempeño académico.

Otra de las variables que se consideran centrales en el desempeño académico de niños, niñas y adolescentes, que ambos conceptos en revisión abordan, es la autoeficacia, definida por Bandura (1997) como la percepción que posee el individuo acerca de sus propias capacidades para desarrollar adecuadamente una tarea específica. De modo similar, Schunk (1991) define la autoeficacia del estudiantado como "juicios” (p. 209) que posee sobre sus capacidades y que le permite finalizar una tarea con éxito y adquirir el conocimiento solicitado. Por tanto, resulta relevante destacar que las percepciones que posea el estudiantado sobre sus capacidades juegan un rol determinante en la ejecución y logro de los resultados académicos.

\subsubsection{Aspectos emocionales}

En el aspecto emocional, hay que señalar que ambos conceptos hacen referencia de que es un factor que condiciona el proceso tanto de enseñanza como de aprendizaje. Dentro del compromiso escolar, se destaca la importancia de los vínculos afectivos que establece el estudiantado con sus pares y docentes e incluso con la tarea en sí misma, haciendo notar que los vínculos afectivos que establece en sus contextos de aprendizaje pueden generarle emociones tanto positivas como negativas que redundarán en sensaciones de satisfacción o insatisfacción con el proceso. De modo que el compromiso escolar considera el componente emocional, en tanto tiene en cuenta las reacciones positivas y negativas que estudiantes experimentan con sus profesorado, sus compañeros y compañeras, las actividades académicas y la escuela (Finn, 1989; Voelkl, 1997).

En la motivación escolar, el factor emocional se analiza a partir del autoconcepto, es decir, la imagen de sí mismo que posee el estudiantado (Shavelson, Hubner y Stanton, 1976). Esta percepción se desarrolla a partir de la influencia e interacción que el alumnado tenga con su entorno como familia, profesorado, pares de clase. García y Musitu (1993) señalan que dicho concepto repercutirá en la motivación y posterior rendimiento. Coincidentemente, Bandura (1977) plantea que el individuo tiene ciertas expectativas de éxito o fracaso en los resultados que pueden alcanzar frente a una determinada tarea, basado en las creencias y valoraciones que tiene de sus propias capacidades, percepción de sí mismo que influirá en su motivación y rendimiento, aun cuando hay que tener en cuenta que el comportamiento estudiantil estará determinado por el autoconcepto que posea en ese momento. 
Según estos argumentos, se evidencia la diferencia entre ambas visiones: motivación y compromiso. La motivación centra su análisis en el autoconcepto que es un proceso interno, que se sustenta en la interpretación que el sujeto hace de las ideas, opiniones y de las interacciones que establece con otros, de modo que se trataría de un proceso más bien psicológico.

En cambio, el compromiso centra su análisis en los vínculos, poniendo énfasis en el componente social de las interacciones que establece el estudiantado con los otros actores del proceso educativo, cobrando importancia el proceso de aprendizaje, como un proceso de construcción colectiva del conocimiento.

\subsubsection{Aspectos de participación}

Dentro del plano de factores de tipo contextual, el compromiso incorpora la variable conductual relacionándola con la participación e involucramiento en actividades académicas, sociales y extracurriculares (Fredricks et al., 2004); en tanto que la motivación considera el componente participación en contexto de otros temas y situaciones que ayuden a valorar el aprendizaje, que le den sentido y donde se rescate la experiencia previa. En este sentido, no hace hincapié en las actividades extracurriculares o en sentido social de las interacciones, sino pone el acento en los aspectos vivenciales y simbólicos de lo colectivo, y en cómo estos dan significado al aprendizaje.

En busca de poder encontrar similares discusiones sobre la distinción y relación de ambos conceptos, nos encontramos que, desde la bibliografía de origen iberoamericano, como ya se ha mencionado, existe escasa mención al concepto de compromiso escolar, a diferencia de un amplio número de investigaciones asociadas a motivación escolar.

Una posible explicación de este hecho podría ser que ambos conceptos hayan sido considerados como sinónimos, pues a la base de la idea de compromiso y sus dimensiones constitutivas, está el uso de la teoría motivacional, lo que podría entenderse compromiso como motivación escolar.

Por otro lado, se podría entender que el constructo de motivación escolar sería entendido como un concepto amplio que abarcaría elementos asociados a compromiso. Sin embargo, desde el análisis realizado de los textos anglosajones, la motivación escolar es un elemento necesario, vital pero no el único para desarrollar compromiso. Por lo tanto, este último concepto tendría un rol más amplio que implicaría, principalmente, la ejecución de una actividad en un contexto determinado.

\section{Conclusiones}

El propósito de este trabajo ha sido intentar ofrecer una síntesis en la comprensión de los conceptos de compromiso y motivación escolar, ha buscado principalmente entregar una diferenciación, incluyendo las dimensiones o componentes que conforman dichos conceptos.

Esta revisión permite dar cuenta de que la bibliografía iberoamericana no exhibe un uso extendido de ambos conceptos como nociones diferenciables entre sí, y aún no se ha planteado una discusión al respecto. Inferimos que al concepto de compromiso escolar no se le ha dado actualmente la importancia necesaria para ser tratado como un constructo independiente en nuestros contextos, sino que se ha tratado como sinónimo de motivación escolar.

Hay suficiente evidencia para considerar que la motivación es un componente sumamente importante que incide en la adaptación y el logro de los aprendizajes estudiantiles. Sin embargo, obviar o no realizar el ejercicio de diferenciarla de compromiso escolar sería estar negando el constructo que permite la interacción directa con el ambiente, es decir, con la tarea a realizar. Por lo tanto, las estrategias de intervención que se pueden implementar en un contexto educativo dado podrían ser de alcance limitado. 
Desde una mirada práctica, la no claridad o diferenciación de ambos conceptos no permitiría construir una base clara y sólida sobre los posibles lineamientos de intervención a trabajar en un contexto educativo. En este sentido, la construcción de programas de apoyo para el mayor involucramiento del estudiante en su aprendizaje debe pasar por considerar las variables o factores claves que lo conforman, ya sea del compromiso o la motivación escolar. Específicamente. tal como lo señalan De Toro et al. (2016), existe un amplio abanico de modelos y prácticas que apuntan a la intervención en el contexto escolar y que presentan evidencia positiva en su ejecución. Sin embargo, la mayoría proviene de modelos educativos anglosajones o de contextos que difieren de las características propias que posee nuestra cultura iberoamericana. Así también estas intervenciones son planteadas desde el compromiso y no de la motivación escolar, por lo tanto, dejan un vació de propuestas de este concepto.

Por otro lado, tanto en motivación como compromiso escolar se hace alusión a los factores contextuales, expresados en vínculos emocionales del estudiantado con los otros actores del proceso educativo, y a la participación como elemento de contexto. En la bibliografía revisada, esta experiencia emocional y relacional del proceso de aprendizaje queda reducida al contexto escolar, se observa que no se otorga la fuerza y protagonismo suficiente a la familia como un elemento contextual significativo y relevante, toda vez que es el espacio de socialización primaria del individuo, pero además la más significativa fuente de apoyo en el desarrollo biopsicosocial de la persona.

Aunque hay algunos estudios que plantean, específicamente, que las motivaciones externas, en especial, familiares y sociales son parte importante de la motivación del estudiantado para el logro de sus metas, y provienen de personas como padres, madres, hermanos, hermanas, profesorado, pares y de circunstancias que le rodean, no está suficientemente considerada su incidencia en el éxito de las trayectorias educativas. Esto, además, representa un desafío en líneas de investigaciones futuras en evaluar la influencia de este componente contextual en los resultados académicos de nuestro estudiantado.

Es posible que la omisión del constructo "compromiso escolar" en laos textos publicados en medios de origen iberoamericano pueda radicar en las cargas semánticas que se asocian a los conceptos al ser traducidos. En este sentido, mientras "motivation" es fácilmente traducible en "motivación", traducir "engagement" a "compromiso" puede ser adecuado para aquellas definiciones del constructo que aludan al sentirse parte de la escuela o el curso (esto es, compromiso a relaciones con el establecimiento u otras personas); pero posiblemente otro vocablo sería más funcional, si buscamos referirnos a la experiencia de fluidez que se experimenta al estar profundamente inmerso cognitiva y afectivamente en una tarea. Por ahora, proponemos que una alternativa para favorecer la claridad conceptual sería lograr mayor especificidad respecto a cada concepto aludido, a modo de ejemplo, al referirse a "compromiso del estudiantado con su escuela", "compromiso del estudiantado con sus pares de curso", o bien "involucramiento del estudiantado con las tareas en matemáticas".

Finalmente, añadimos que sería necesario una revisión amplia de cómo las diversas descripciones de ambos conceptos y cómo sus sobreposiciones tienen posibles efectos sobre aspectos metodológicos (es decir, cómo se están midiendo estos constructos según sus diferentes definiciones) que finalmente son aquellos que permiten levantar evidencia a favor o en contra de la relevancia que pueda tener cualquier variable que confluya en el proceso educativo.

\section{ReFERENCIAS}

Alderman, M. (2008). Motivation for achievement: Possibilities for teaching and learning (3a ed.). Nueva York: Taylor and Francis.

Appleton, J., Christenson, S., \& Furlong, M. (2008). Student engagement with school: Critical conceptual and methodological issues of the construct. Psychology in the Schools, 45(5), 369-386. doi: https://doi.org/10.1002/ pits. 20303 
María José SANDOVAL-Muñoz, et AL. Compromiso y MOTIVACión ESCOLAR: UNa discusión CONCEPTUAL ${ }^{1}$

Archambault. J. y Chouinard, R. (1996). Vers une gestion éducative de la clase. Montréal: Morin.

Asensio, J. (1994). Hijos con fracaso escolar. Barcelona: Grupo Editorial CEAC.

Bandura, A. (1977). Self-efficacy: Toward a unifying theory of behavioral change. Psychological Review, 84(2), 191-215. doi: https://doi.org/10.1037/0033-295X.84.2.191

Bandura, A. (1997). Self-efficacy. The exercise of control. Nueva York: Freeman.

Beltrán, J. (1993). Procesos, estrategias y técnicas de aprendizaje. Madrid: Síntesis.

Broc, M. Á. (2000). Autoconcepto, autoestima y rendimiento académico en alumnos de $4^{\circ}$ de E.S.O.: Implicaciones psicopedagógicas en la orientación y tutoría. Revista de Investigación Educativa, 18(1), 119-146. Recuperado de http://revistas.um.es/rie/article/view/121521/114201

Bueno, J. A. (1995). Motivación y aprendizaje. En J. Beltrán y J. A. Bueno (Eds.), Psicología de la educación (pp. 227-255). Barcelona: Marcombo.

Christenson, S., Reschly, A., \& Wylie, C. (2012). Handbook of research on student engagement. New York: Springer. doi: https://doi.org/10.1007/978-1-4614-2018-7

Corso, M., Bundick, M., Quaglia, R. \& Haywood, D. (2013). Where student, teacher, and content meet: Student engagement in the secondary school classroom. American Secondary Education, 41(3), 50-61.

Covington, M. (2000) Goal Theory, motivation and school achievement: an integrative review. Annual Review of Psychology, 51, 171-200. doi: https://doi.org/10.1146/annurev.psych.51.1.171

Csikszentmihalyi, M. (1990). Flow: The psychology of optimal performance. NY: Cambridge University Press.

De Toro, X., Sandoval, M. J., Trigger, J., Saracostti, M., Viveros, J., Riquelme, S., ... Navarro, J. J. (2016). Manual de intervenciones sobre el compromiso escolar: Una variable clave para predecir procesos de desescolarización. Colección Ciencias Sociales. Santiago de Chile: Ediciones Universidad de la Frontera.

Dotterer, A., McHale, S., y Crouter, A. (2007). Implications of out-of-school activities for school engagement in African American adolescents. Journal of Youth and Adolescence, 36(4), 391-401. doi: https:// doi.org/10.1007/s10964-006-9161-3

Faria, C., Freire, S., Galvão, C., Reis, P., y Baptista, M. (2012). Students at risk of dropping out: how to promote their engagement with school science? Science Education International, 23(1), 20-39. Recuperado de http:// www.icaseonline.net/sei/march2012/p2.pdf

Finn, J. D. (1989). Withdrawing from school. Review of educational research. 59(2), 117-142. doi: https:// doi.org/10.3102/00346543059002117

Finn, J. y Voelkl, K. (1993). School characteristics related to student engagement. The Journal of Negro Education, 62(3), 249-268. doi: https://doi.org/10.2307/2295464

Fredricks, J. A., Blumenfeld, P. C. y Paris, A. H. (2004). School engagement: Potential of the concept, state of the evidence. Review of educational research, 74(1), 59-109. doi: https://doi.org/10.3102/00346543074001059

Furlong, M., y Christenson, S. (2008). Engaging students at school and with learning: A relevant construct for all students. Psychology in the Schools, 45(5), 365-368. doi: https://doi.org/10.1002/pits.20302

Furrer, C. y Skinner, E. (2003). Sense of relatedness as a factor in children's academic engagement and performance. Journal of Educational Psychology, 95(1), 148-162. doi: https://doi.org/10.1037/0022-0663.95.1.148

García, F. J. y Doménech, F. (1997). Motivación, aprendizaje rendimiento escolar. Reme, 1(0), 1-36.

García, F. J. y Musitu, G. (1993). Rendimiento académico y autoestima en el Ciclo Superior de EGB. Revista de Psicología de la Educación, 4(11), 73-87.

García, M. A., Barbero M. I., Ávila, I. y García. M. C. (2003). La motivación laboral de los jóvenes en su primer empleo. Psicothema, 15(1), 109-113. Recuperado de http://www.psicothema.es/pdf/1031.pdf

Godefroid, J. (1991). Psychologie. Science humaine et science cognitive. Montreal: Éditions HRW.

Green, G., Rhodes, J., Hirsch, A., Suárez-Orozco, C. \& Camic, P. (2008). Supportive adult relationships and the academic engagement of Latin American immigrant youth. Journal of School Psychology, 46(4), 393-412. doi: https://doi.org/10.1016/j.jsp.2007.07.001 
Hart, S., Stewart, K. \& Jimerson, S. (2011). The student engagement in schools questionnaire (SESQ) and the teacher engagement report form-new (TERF-N): Examining the preliminary evidence. Contemporary School Psychology, 15(1), 67-79. Recuperado de https://files.eric.ed.gov/fulltext/EJ934707.pdf

Hattie, J. (2013). Visible learning: A synthesis of over 800 meta-analyses relating to achievement. New York: Routledge.

Hazel, C., Vazirabadi, G., \& Gallagher, J. (2013). Measuring aspirations, belonging, and productivity in secondary students: Validation of the student school engagement measure. Psychology in the Schools, 50(7), 689-704. doi: https://doi.org/10.1002/pits.21703

Hickey, D. (2003). Engaged participation versus marginal nonparticipation: A stridently Stridently sociocultural approach to acheivement motivation. The Elementary School Journal, 103(4), 401-429. doi: https:// doi.org/10.1086/499733

Inglés, C., García-Fernández, J., Castejón, J., Valle, A., Delgado, B. \& Marzo, J. (2009). Reliability and validity evidence of scores on the achievement goal tendencies questionnaire in a sample of Spanish students of compulsory secondary education. Psychology in the Schools, 46(10), 1048-1060. doi: https://doi.org/10.1002/pits.20443

Jimerson, S., Campos, E. \& Greif, J. (2003). Toward an understanding of definitions and measures of school engagement and related terms. The California School Psychologist, 8(1), 7-27. doi: https://doi.org/10.1007/ BF03340893

Legendre, R. (1993). Dictionnaire actuel de l'éducation. Montreal: Guérin.

Maehr, M. y Meyer, H. (1997). Understanding motivation and schooling: Where we've been, where we are, and where we need to go. Educational Psychology Review, 9(4),371-409. doi: https://doi.org/10.1023/A:1024750807365

Marjoribanks, K. (2003). Family background, adolescent's achievement and aspirations, and young adults's enrolment in Australian Universities. Aula Abierta, 82, 147-159.

Marks, H. (2000). Student engagement in instructional activity: Patterns in the elementary, middle, and high school years. American educational research journal, 37(1), 153-184. doi: https:// doi.org/10.3102/00028312037001153

Martínez, J. A (2011a). Automotivación y rendimiento académico en el espacio europeo de educación superior. Cuadernos de Educación y Desarrollo, 3(28). Recuperado de http://www.eumed.net/rev/ced/28/jamg.htm? $\mathrm{cv}=1$

Martínez, J. A. (2011b). Diseño y validación de cuestionario de automotivación AM-24 en el marco del Espacio Europeo de Educación Superior. Cuadernos de Educación y Desarrollo, 3(27), 1-12. Recuperado de http:// www.eumed.net/rev/ced/27/jamg.pdf

McClelland, D. (1989). Motivational factors in health and illness. American Psychologist, 44(4), 675-683. doi: https://doi.org/10.1037/0003-066X.44.4.675

Meece, J., Anderman, E. y Anderman, L. (2006). Classroom goal structure, student motivation, and academic achievement. American Review of Psychology, 57, 487-503. doi: https://doi.org/10.1146/ annurev.psych.56.091103.070258

Newmann, F., Wehlage, G. y Lamborn, S. (1992). The significance and sources of student engagement. En F. Newman (Ed.), Student engagement and achievement in American secondary schools (pp. 11-39). New York: Teachers College Press.

Núñez, J. y González-Pumariega, S. (1996). Motivación y aprendizaje escolar. Actas Congreso Nacional sobre Motivación e Instrucción (pp. 53-72). Congreso llevado a cabo en Coruña, España.

Peralbo, M., Sánchez, J. y Simón, M. (1986). Motivación y aprendizaje escolar: Una aproximación desde la teoría de la autoeficacia. Revista Infancia y Aprendizaje, 9(35-36), 37-45. doi: https:// doi.org/10.1080/02103702.1986.10822127

Pintrich, P., Schunk, D. y Luque, M. (2006). Motivación en contextos educativos: teoría, investigación y aplicaciones. Madrid: Pearson Educación.

Pintrich, P. R. y De Groot, E. V. (1990). Motivational and self-regulated learning components of classroom performance. Journal of Educational Psychology, 82, 33-40. doi: https://doi.org/10.1037/0022-0663.82.1.33 
María José SANDOVAL-Muñoz, et AL. Compromiso y MOTIVACión ESCOLAR: UNa discusión CONCEPTUAL ${ }^{1}$

Raynal, F. y Rieunier, A. (2014). Pédagogie, dictionnaire des concepts clés: Apprentissage, formation, psychologie cognitive. Issy-les-Moulineaux: ESF.

Robson, K., Albanese, P., Harrison, D. y Sanders, C. (2014). School Engagement among Youth in Canadian Forces Families: A Comparative Analysis. Journal of Educational Research, 59(3), 363-381.

Russell, V. J., Ainley, M., y Frydenberg, E. (2005) Student motivation and engagement. Schooling issues digest, 2.

Schunk, D. (1991). Self-efficacy and academic motivation. Educational psychologist, 26(3-4), 207-231. doi: https:// doi.org/10.1080/00461520.1991.9653133

Shavelson, R. J., Hubner, J. J., y Stanton, G. C. (1976). Self-concept: Validation of construct interpretations. Review of Educational Research, 46(3), 407-441. doi: https://doi.org/10.3102/00346543046003407

Shernoff, D. (2013). Optimal learning environments to promote student engagement. New York: Springer. doi: https://doi.org/10.1007/978-1-4614-7089-2

Shernoff, D. y Schmidt, J. (2008). Further evidence of an engagement-achievement paradox among US high school students. Journal of Youth and Adolescence, 37(5), 564-580. doi: https://doi.org/10.1007/s10964-007-9241-z

Skinner, E., Kindermann, T., Connell, J., y Wellborn, J. (2009). Engagement and disaffection as organizational constructs in the dynamics of motivational development. En K. Wentzel y A. Wigfield (Eds.), Handbook of motivation at school (pp. 223-245). New York: Routledge.

Steinmayr, R. y Spinath, B. (2009). The importance of motivation as a predictor of school achievement. Learning and Individual Differences, 19(1), 80-90. doi: https://doi.org/10.1016/j.lindif.2008.05.004

Tardif, J. (1992). Pour un enseignement stratégique. Montreal: Éditions Logiques.

Torío, S. (2004). Familia, escuela y sociedad. Aula Abierta, 83, 35-52. Recuperado de https://dialnet.unirioja.es/ servlet/articulo?codigo $=1173765$

Torrano, F. y González, M. C. (2004). El aprendizaje autorregulado: Presente y futuro de la organización. Electronic journal of research in educational psychology, 2(1), 1-34. Recuperado de http://www.investigacionpsicopedagogica.org/revista/new/ContadorArticulo.php?27

Valle, A. y González, R. (1996). Una aproximación teórica al concepto de metas académicas y su relación con la motivación escolar. Psicothema, 8(1), 45-56. Recuperado de http://www.redalyc.org/pdf/727/72780104.pdf

Valle, A., Núñez, J., Rodríguez, S., Cabanach, R., González-Pienda, J. y Rosario, P. (2010). Perfiles motivacionales y diferencias en variables afectivas, motivacionales y de logro. Universitas Psychologica, 9(1), 109-121. Recuperado de http://revistas.javeriana.edu.co/index.php/revPsycho/article/view/283/427

Veiga, F. (2012). Proposal to the PISA of a new scale of students' engagement in school. Procedia-Social and Behavioral Sciences, 46, 1224-1231. doi: https://doi.org/10.1016/j.sbspro.2012.05.279

Veiga, F. (2013). Envolvimento dos alunos na escola: Elaboração de uma nova escala de avaliação. International Journal of Developmental and Educational Psychology, 1(1), 441-450. Recuperado de http://repositorio.ul.pt/ bitstream/10451/10032/1/EAE_nova\%20escala_\%20Moscovo\%202013.pdf

Viau, R. (1997). La motivation en contexte scolaire. Bruxelles: De Boeck.

Voelkl, K. (1997). Identification with school. American Journal of Education, 105(3), 294-318. doi: https:// doi.org/10.1086/444158

Weiss, C., Carolan, B. \& Baker-Smith, E. (2010). Big school, small school: (Re) testing assumptions about high school size, school engagement and mathematics achievement. Journal of Youth and Adolescence, 39(2), 163-176. doi: https://doi.org/10.1007/s10964-009-9402-3

Wentzel, K. (2005). Peer relationships, motivation, and academic performance at school. In Elliot, J. y Dweck, C. (Eds.), Handbook of Competence and Motivation (pp. 279-296). Nueva York: Guilford Press.

Wentzel, K., Wigfield, A. \& Miele, D. (2009). Handbook of motivation at school. New York: Routledge.

Zimmerman, B. J. (1998). Academic studing and the development of personal skill: A self-regulatory perspective. Educational psychologist, 33(2-3), 73-86. doi: https://doi.org/10.1080/00461520.1998.9653292

Zimmerman, P. (1999). Autoeficacia y desarrollo educativo. En A. Bandura (Ed.), Auto-eficacia: Cómo afrontamos los cambios de la sociedad actual. España: Desclée De Brouwer. 


\section{Notas}

[1] El desarrollo del presente trabajo forma parte del proyecto CONICYT-FONDEF-ID14I10078

\section{BY-NC-ND}

